\title{
Regular black holes with a nonlinear electrodynamics source
}

\author{
Leonardo Balart ${ }^{1 *}$ and Elias C. Vagenas $^{2+}$ \\ 1 Departamento de Ciencias Físicas, Facultad de Ingeniería y Ciencias, \\ Universidad de La Frontera, Casilla 54-D, Temuco, Chile and \\ 2 Theoretical Physics Group, Department of Physics, \\ Kuwait University, P.O. Box 5969, Safat 13060, Kuwait \\ *email:leonardo.balart@ufrontera.cl \\ ${ }^{+}$email: elias.vagenas@ku.edu.kw
}

\begin{abstract}
We construct several charged regular black hole metrics employing mass distribution functions which are inspired by continuous probability distributions. Some of these metrics satisfy the weak energy condition and asymptotically behave as the ReissnerNordström black hole. In each case, the source to the Einstein equations corresponds to a nonlinear electrodynamics model, which in the weak field limit becomes the Maxwell theory (compatible with the Maxwell weak field limit or approximation). Furthermore, we include other regular black hole solutions that satisfy the weak energy condition and some of them correspond to the Maxwell theory in the weak field limit.
\end{abstract}

\section{Introduction}

Charged regular black holes are solutions of Einstein equations that have horizons and, contrary to Reissner-Nordström black holes which have singularities at the origin, their metrics as well as their curvature invariants $R, R_{\mu \nu} R^{\mu \nu}, R_{\kappa \lambda \mu \nu} R^{\kappa \lambda \mu \nu}$ are regular everywhere [1]. This type of black holes violates the strong energy condition somewhere in the spacetime (see, e.g., Ref. [2] or Ref. [3]); however, some of these solutions satisfy the weak energy condition (WEC) everywhere. Those that satisfy the WEC necessarily have a de Sitter center [4]. In addition, there are other features that characterize regular black holes which are due to the nonlinearities of the field equations. For instance, the thermodynamic quantities of these black holes do not satisfy the Smarr formula (see, e.g., Ref. [5]), the identity of BoseDadhich [6] refers to the relation between the Brown-York energy, and the Komar charge is not satisfied by regular black holes [7]. 
Several regular black hole solutions have been found by coupling gravity to nonlinear electrodynamics theories (for a more detailed review see Ref. [1 and references cited therein). Two of these solutions satisfy the WEC and asymptotically behave as the ReissnerNordström black hole; one of them was reported by Ayón-Beato and García in Ref. [8] (see also Ref. [9] which is a generalization of this case) and the other one was presented by Dymnikova in Ref. [10]. Additionally Ayón-Beato and García obtained other solutions in Refs. [11] and [12] (see also Ref. [13]), which asymptotically behave as the Reissner-Nordström solution but do not satisfy the WEC. These authors also considered the Bardeen regular black hole [14], which satisfies the WEC but asymptotically does not behave as the Reissner-Nordström solution, and interpreted it as gravity coupled to a theory of nonlinear electrodynamics for a self-gravitating magnetic monopole in Ref. 15. Recently, the authors of this paper have presented a family of regular black hole metrics in Ref. [16], which by construction satisfy the WEC. Other black hole solutions that satisfy the WEC have been found by considering a Gaussian distribution for the mass density in Ref. [17] and also for both the mass density and the charge density in Ref. [18]. A noncharged black hole solution which is also regular and satisfies WEC was given by Dymnikova in Ref. [19. A black hole solution similar to the Bardeen solution, i.e., one that satisfies the WEC but asymptotically does not behave as the Reissner-Nordström solution, is given by Hayward in Ref. [20]. Other solutions of regular black holes have been obtained by the junction of two spherically symmetric regions in Refs. [2] and [21]. In particular, Ref. [2] carried out a detailed analysis of the energy conditions of these solutions, and the authors showed that not all cases verify the WEC everywhere. The solutions considered in Ref. [2] satisfy the WEC, where the outer and the inner regions are separated by an electrically charged spherically symmetric coat and the outer region behaves as a Reissner-Nordström solution.

Using some of these examples, several articles have discussed some other features of this family of solutions or the particularities that differentiate this family of solutions with respect to Reissner-Nordström solutions. In Ref. [22], the Bardeen black hole was considered as a gravitational lens, and the results were compared with those obtained by a solution of Schwarzschild type. The thermodynamics and evaporation process of the solution proposed by Hayward are discussed in Ref. [23]. The solution given in Ref. [8] has been considered with a cosmological constant in Ref. [24], both the thermodynamics and stability have been analyzed. There are various studies which consider the regular black hole defined by Ref. [11]. In particular, the thermodynamics of this solution was studied in Ref. [25], and the entropy of the corresponding extremal case was studied in Refs. [26], [27], and [28]. It was also used to construct a regular black hole solution in an asymptotically de Sitter universe in Ref. [29]. Likewise it was used for construction of the renormalized stress-energy tensor for the massive scalar fields case [30, 31] and for the massive spinor and vector fields cases [32. It was also used in the context of quadratic gravity in Refs. [33] and [34. Other studies have used two or more of the solutions of regular black holes mentioned in the previous paragraph: for the construction of gravastar models in Ref. [35] and for obtaining solutions in $f(R)$ modified theories of gravity in Ref. [36]. The stability of some solutions was studied in Refs. [37], [38, and 39 .

In this work we construct several charged regular black hole metrics in the context of 
theories with nonlinear electrodynamics coupled to general relativity. The mass functions are inspired by continuous probability distributions. Some of these metrics satisfy the WEC and asymptotically behave as the Reissner-Nordström black hole.

This paper is organized as follows. In Sec. 2, we construct a general regular black hole metric for mass distribution functions that are inspired by continuous probability distributions. We also construct the corresponding electric field for each black hole solution in terms of a general mass distribution function. In Sec. 3, we present two examples of black hole solutions employing the methodology developed in Sec. 2. In Sec. 4, we generalize the construction presented in Sec. 2 by considering distribution functions raised to the power of a real number greater than zero. Moreover, we give two examples of black hole solutions by using this generalized construction. In Sec. 5, we consider a particular distribution in order to obtain a regular black hole metric, and then find the conditions under which the WEC is satisfied. In Sec. 6, we present some more regular black hole metrics that satisfy the WEC and, with the exception of one case, in the weak field limit the corresponding black hole solutions do not describe the Maxwell theory . In Sec. 7, we present regular black hole metrics that asymptotically behave as the Reissner-Nordström solution and some satisfy the WEC. Finally, in Sec. 8, we briefly summarize our results. In Appendix A, we give some probability distributions, and in Appendix B, we give a brief description of the dual $\mathrm{P}$ formalism.

\section{General considerations}

We consider the line element for the most general static and spherically symmetric metric,

$$
d s^{2}=-f(r) d t^{2}+f(r)^{-1} d r^{2}+r^{2}\left(d \theta^{2}+\sin ^{2} \theta d \phi^{2}\right)
$$

with

$$
f(r)=1-\frac{2 m(r)}{r}
$$

and where $m(r)$ is the mass function. The outer and the inner horizons are located at $r_{+}$ and $r_{-}$, respectively, satisfying $r_{ \pm}=2 m\left(r_{ \pm}\right)$.

To construct metrics of regular black holes where the invariant scalars and the electric fields are regular everywhere, we express the mass function $m(r)$ as

$$
m(r)=\frac{\sigma(r)}{\sigma_{\infty}} M
$$

where the distribution function $\sigma(r)$ satisfies $\sigma(r)>0$ and $\sigma^{\prime}(r)>0$ for $r \geq 0$. Additionally, $\sigma(r) / r \rightarrow 0$ as $r \rightarrow 0$ and $\sigma_{\infty}=\sigma(r \rightarrow \infty)$ represents the normalization factor. We will employ distribution functions inspired by the shape of probability density functions that satisfy $\sigma(x)>0, \sigma^{\prime}(x)<0$ for $x \geq 0$ and $\sigma^{\prime}(x=0) \neq 0$ (see Appendix A). Moreover, depending on the choice of the distribution function, the variable $x$ is replaced in $\sigma(x)$ by 


\begin{tabular}{|c|}
\hline Distribution function $\sigma(r)$ \\
\hline $\exp \left(-\frac{q^{2}}{2 M r}\right)$ \\
\hline$\left(\exp \left(\frac{q^{2}}{M r}\right)+1\right)^{-1}$ \\
\hline $\exp \left(-\sqrt{\frac{2 q^{2}}{M r}}\right) /\left(1+\exp \left(-\sqrt{\frac{2 q^{2}}{M r}}\right)\right)^{2}$ \\
\hline $2\left(\exp \left(\sqrt{\frac{q^{2}}{M r}}\right)+\exp \left(-\sqrt{\frac{q^{2}}{M r}}\right)\right)^{-1}$ \\
\hline$\left(\frac{q^{2}}{M r}\right) /\left(\exp \left(\frac{q^{2}}{M r}\right)-1\right)$ \\
\hline$\left(\frac{6 q^{2}}{M r}\right) \exp \left(\sqrt{\frac{6 q^{2}}{M r}}\right) /\left(\exp \left(\sqrt{\frac{6 q^{2}}{M r}}\right)-1\right)^{2}$ \\
\hline
\end{tabular}

Table 1: Examples of probability distribution functions used in the mass functions of regular black holes.

$1 / r$ or $1 / \sqrt{r}$ with appropriate factors which depend on the mass $M$ and the charge $q$ of the regular black hole.

The distribution functions are listed in Table 1 in the same order as in Appendix A and satisfy the condition

$$
\frac{m(r)}{r} \rightarrow 0 \quad \text { when } \quad r \rightarrow 0
$$

and asymptotically approach the Reissner-Nordström metric

$$
f(r)=1-\frac{2 m(r)}{r} \approx 1-\frac{2 M}{r}+\frac{q^{2}}{r^{2}} .
$$

For each regular black hole solution of which the mass function is given by Eq. (3), we can obtain the electric field by considering the components of the Einstein gravitational field equations $G_{\mu \nu}=8 \pi T_{\mu \nu}$. Here, the energy-momentum tensor $T_{\mu \nu}$ is given as

$$
T_{\mu \nu}=L(F) g_{\mu \nu}-L_{F} F_{\mu \alpha} F_{\nu}^{\alpha}
$$

where the Lagrangian $L(F)$ depends on the Lorentz invariant $F=\frac{1}{4} F^{\mu \nu} F_{\mu \nu}$ and we have defined $L_{F}=d L / d F$.

If we restrict to the electric field, i.e., we consider $F_{\mu \nu}=E(r)\left(\delta_{\mu}^{0} \delta_{\nu}^{1}-\delta_{\mu}^{1} \delta_{\nu}^{0}\right)$, and if we employ Eqs. (2) and (3) given here as well as Eq. (7) from Ref. [16], then we can write the components of $G_{\mu \nu}$ as

$$
\begin{aligned}
& G_{0}^{0}=G_{1}^{1}=-\frac{2 M}{r^{2}} \frac{\sigma^{\prime}(r)}{\sigma_{\infty}}=8 \pi\left(L(F)+E^{2} L_{F}\right) \\
& G_{2}^{2}=G_{3}^{3}=-\frac{M}{r} \frac{\sigma^{\prime \prime}(r)}{\sigma_{\infty}}=8 \pi L(F) .
\end{aligned}
$$


In addition, the electromagnetic field equations $\nabla_{\mu}\left(F^{\mu \nu} L_{F}\right)=0$ imply

$$
E(r) L_{F}=-\frac{q}{4 \pi r^{2}}
$$

By subtracting Eq. (7) from Eq. (8) and using Eq. (9), we obtain a general expression for the electric field $E$ of the regular black hole solutions

$$
E(r)=-\frac{r^{3}}{2 q} \frac{M}{\sigma_{\infty}} \frac{d}{d r}\left(\frac{1}{r^{2}} \frac{d \sigma(r)}{d r}\right)
$$

After considering any one of the distribution functions listed in Table 1, the corresponding electric fields are regular everywhere and asymptotically behave as

$$
E(r) \approx \frac{q}{r^{2}}
$$

as expected from Eq. (15).

It is important to note that the distribution function $\sigma(r)$, which is associated with the mass function, converges to zero approximately as $e^{-\frac{1}{r}}$ when $r \rightarrow 0$, and the same applies to the derivatives of any order of $\sigma(r)$. Therefore, the curvature invariants, namely the Ricci scalar

$$
R=\frac{2 M}{r^{2} \sigma_{\infty}}\left(2 \sigma^{\prime}(r)+r \sigma^{\prime \prime}(r)\right)
$$

the Ricci squared

$$
R_{\mu \nu} R^{\mu \nu}=\frac{2 M^{2}}{r^{4} \sigma_{\infty}^{2}}\left(4 \sigma^{\prime}(r)^{2}+r^{2} \sigma^{\prime \prime}(r)^{2}\right)
$$

and the Kretschmann scalar

$$
\begin{aligned}
R_{\kappa \lambda \mu \nu} R^{\kappa \lambda \mu \nu}= & \frac{4 M^{2}}{r^{6} \sigma_{\infty}^{2}}\left[4\left(3 \sigma(r)^{2}-4 r \sigma(r) \sigma^{\prime}(r)+2 r^{2} \sigma^{\prime}(r)^{2}\right)\right. \\
& \left.+4 r^{2}\left(\sigma(r)-r \sigma^{\prime}(r)\right) \sigma^{\prime \prime}(r)+r^{4} \sigma^{\prime \prime}(r)^{2}\right]
\end{aligned}
$$

are regular everywhere.

An equivalent description could be given by using the dual $\mathrm{P}$ formalism implemented in Ref. [40] (some details of this formalism are presented in Appendix B).

\section{Two examples of regular black hole metrics}

In this section, we consider two of the distribution functions listed in Table 1 in order to construct regular black hole metrics. 1

First, we consider the exponential distribution, where the variable $x$ is replaced with $q^{2} /(2 M r)$ in Eq. (68) of Appendix A (see also Table 1), and we obtain

\footnotetext{
${ }^{1} \mathrm{~A}$ similar treatment can also be applied to the rest of the distribution functions in Table 1.
} 


$$
\sigma(r)=\exp \left(-\frac{q^{2}}{2 M r}\right)
$$

where the normalization factor is

$$
\sigma_{\infty}=1
$$

Thus, the metric function is of the form

$$
f(r)=1-\frac{2 M}{r} \exp \left(-\frac{q^{2}}{2 M r}\right)
$$

which vanishes at the location of the horizons, i.e., $r_{ \pm}$. By solving the resulting equation, namely $f\left(r_{ \pm}\right)=0$, we find the real roots for $r_{ \pm}$,

$$
r_{+}=-\frac{q^{2}}{2 M W\left(0,-\frac{q^{2}}{4 M^{2}}\right)} \quad \text { and } \quad r_{-}=-\frac{q^{2}}{2 M W\left(-1,-\frac{q^{2}}{4 M^{2}}\right)}
$$

where $W$ is Lambert's $W$ function.

The corresponding electric field can be directly obtained from Eq. (10)

$$
E(r)=\frac{q}{r^{2}}\left(1-\frac{q^{2}}{8 M r}\right) \exp \left(-\frac{q^{2}}{2 M r}\right)
$$

which is regular everywhere and asymptotically behaves as $E(r)=q / r^{2}+O\left(1 / r^{3}\right)$. It should be noted that in this example we have the extremal regular black hole when $|q|=1.213 \mathrm{M}$. Second, we consider the Fermi-Dirac-type distribution. Thus, if $x$ is replaced with $q^{2} /(M r)$ in Eq. (69) of Appendix A (see also Table 1), we obtain the distribution function

$$
\sigma(r)=\frac{1}{\exp \left(\frac{q^{2}}{M r}\right)+1}
$$

with normalization factor

$$
\sigma_{\infty}=1 / 2
$$

and the metric function is now written as

$$
f(r)=1-\frac{2 M}{r}\left(\frac{2}{\exp \left(\frac{q^{2}}{M r}\right)+1}\right) .
$$

It is noteworthy that this metric function corresponds to an Ayón-Beato and García black hole [11]. In addition, this metric function satisfies the equation $f\left(r_{ \pm}\right)=0$ for the location of the horizons, which now reads

$$
r_{ \pm}=2 M\left(\frac{2}{\exp \left(\frac{q^{2}}{M r_{ \pm}}\right)+1}\right)
$$


This equation has two real roots which again will be expressed in terms of Lambert's W function [30]:

$$
r_{+}=-\frac{4 q^{2}}{4 M W\left(0,-\frac{q^{2}}{4 M^{2}} e^{\frac{q^{2}}{4 M^{2}}}\right)-\frac{q^{2}}{M}} \quad \text { and } \quad r_{-}=-\frac{4 q^{2}}{4 M W\left(-1,-\frac{q^{2}}{4 M^{2}} e^{\frac{q^{2}}{4 M^{2}}}\right)-\frac{q^{2}}{M}} .
$$

Employing Eq. (10), the corresponding electric field is now written as

$$
E(r)=\frac{q}{r^{2}} \operatorname{sech}^{2}\left(\frac{q^{2}}{2 M r}\right)\left(1-\frac{q^{2}}{4 M r} \tanh \left(\frac{q^{2}}{2 M r}\right)\right) .
$$

Furthermore, the corresponding extremal regular black hole is obtained when the value of the charge is $|q|=1.055 M$ (see Refs. [26], [27], and [28]).

\section{More regular black hole metrics}

The use of the distribution functions mentioned above in order to obtain regular black hole solutions can be extended by considering the metric function to take the form

$$
f(r)=1-\frac{2 M}{r}\left(\frac{\sigma(\beta r)}{\sigma_{\infty}}\right)^{\beta}
$$

where the function $\sigma(\beta r)$ corresponds to any one of the mass functions listed in Table 1, but with an additional factor $\beta>0$ for the variable $r$.

In this case, we can see that the mass function satisfies the condition

$$
m(r)=M\left(\frac{\sigma(\beta r)}{\sigma_{\infty}}\right)^{\beta} \rightarrow M \quad \text { when } \quad r \rightarrow \infty
$$

in the same way as the distribution function satisfies

$$
\frac{\sigma(r)}{\sigma_{\infty}} \rightarrow 1 \quad \text { when } \quad r \rightarrow \infty
$$

Furthermore, using the same argument as above, one can find that all curvature invariants are regular.

To illustrate these cases, let us consider here two examples. The first one is constructed by employing the Fermi-Dirac distribution function. In this case, the metric function is written as

$$
f(r)=1-\frac{2 M}{r}\left[\frac{2}{\exp \left(\frac{q^{2}}{\beta M r}\right)+1}\right]^{\beta} .
$$

The outer and the inner horizons of this metric function can be found numerically for each value of $\beta$. The corresponding electric field can be obtained by using Eq. (10); thus 


\begin{tabular}{|c|c|}
\hline$\beta$ & Extremal case \\
\hline 0.5 & $q \approx 0,991 M$ \\
\hline 0.7 & $q \approx 1.023 M$ \\
\hline 1 & $q \approx 1.055 M$ \\
\hline 2.4 & $q \approx 1.124 M$ \\
\hline 4 & $q \approx 1.153 M$ \\
\hline 7 & $q \approx 1.175 M$ \\
\hline 10 & $q \approx 1.186 M$ \\
\hline 100 & $q \approx 1.210 M$ \\
\hline
\end{tabular}

Table 2: Values of $\beta$ and the corresponding charges for the case of an extremal regular black hole when we consider the metric function given by Eq. (29).

$$
\begin{aligned}
E(r) & =\frac{q}{r^{2}} \exp \left(\frac{(1-\beta) q^{2}}{2 \beta M r}\right)\left[\operatorname{sech}\left(\frac{q^{2}}{2 \beta M r}\right)\right]^{1+\beta} \\
& \times\left[1-\frac{q^{2}}{4 M r} \tanh \left(\frac{q^{2}}{2 \beta M r}\right)+\frac{1}{4 \beta M r}\left(\frac{1-\beta}{\exp \left(\frac{q^{2}}{\beta M r}\right)+1}\right)\right] .
\end{aligned}
$$

If we set $\beta \rightarrow 0$ in Eq. (29), then we obtain the metric

$$
f(r)=1-\frac{2 M}{r} \exp \left(-\frac{q^{2}}{M r}\right),
$$

while when we set $\beta \rightarrow \infty$, we reproduce the metric function given by Eq. (17), i.e.,

$$
f(r)=1-\frac{2 M}{r} \exp \left(-\frac{q^{2}}{2 M r}\right)
$$

It should be noted the difference in a factor of 2 between the exponents of Eqs. (31) and (32). In Table 2, we list some values of $\beta$ and the corresponding charges in order to construct the extremal regular black hole metric for this example.

The second example is given by using the logistic distribution function for which the metric function reads

$$
f(r)=1-\frac{2 M}{r}\left[\frac{4 \exp \left(-\sqrt{\frac{2 q^{2}}{\beta M r}}\right)}{\left(1+\exp \left(-\sqrt{\frac{2 q^{2}}{\beta M r}}\right)\right)^{2}}\right]^{\beta} .
$$

As before the horizons can be found numerically for each value of $\beta$. Moreover, the corresponding electric field can be obtained using Eq. (10): 


$$
\begin{aligned}
E(r) & =\frac{q}{r^{2}} \frac{1}{8}\left[\operatorname{sech}\left(\sqrt{\frac{q^{2}}{2 \beta M r}}\right)\right]^{2(1+\beta)} \\
& \times\left[(1+\beta)-\beta \cosh \left(\sqrt{\frac{2 q^{2}}{\beta M r}}\right)+7 \sqrt{\frac{\beta M r}{2 q^{2}}} \sinh \left(\sqrt{\frac{2 q^{2}}{\beta M r}}\right)\right] .
\end{aligned}
$$

At this point a number of comments is in order. First, it is noteworthy that the above expression for the electric field as well as the electric field given by Eq. (30) asymptotically behave as $E=q / r^{2}$. Second, if we set $\beta \rightarrow 0$ in the metric function given by Eq. (33), then we recover the Schwarzschild black hole

$$
f(r)=1-\frac{2 M}{r}
$$

while if we set $\beta \rightarrow \infty$, then we get the metric function given by Eq. (17):

$$
f(r)=1-\frac{2 M}{r} \exp \left(-\frac{q^{2}}{2 M r}\right) .
$$

Third, it should be stressed that in the limit $\beta \rightarrow \infty$ all regular black hole metrics constructed by employing Eq. (26) and the distribution functions listed in Table 1 satisfy the condition

$$
1-\frac{2 M}{r}\left(\frac{\sigma(\beta r)}{\sigma_{\infty}}\right)^{\beta} \rightarrow 1-\frac{2 M}{r} \exp \left(-\frac{q^{2}}{2 M r}\right) .
$$

Finally, when we set $\beta \rightarrow 0$, for some of the cases, we recover the Schwarzschild black hole as is the case for the metric function given by Eq. (33), while for other cases we obtain a black hole metric as the one given by Eq. (31). The only exception here is the regular black hole metric for which we have used the exponential distribution function, namely Eq. (15), for its construction.

\section{Regular black hole metric that satisfies the WEC}

In this section, we consider a distribution function different from those listed in Appendix A (and thus in Table 1). This new distribution function is the log-logistic distribution, which is given by

$$
\sigma(x)=\frac{1}{(1+x)^{2}}
$$

As in the previous section, we will employ the metric function given in Eq. (26). Thus, making the variable change $x \rightarrow 1 / r$ with the appropriate factors, we can write the metric function as 


$$
f(r)=1-\frac{2 M}{r}\left(\frac{1}{\left(1+\frac{q^{2}}{4 \beta M r}\right)^{2}}\right)^{\beta} .
$$

Note that for this metric function, if we set $\beta \rightarrow 0$, then we recover the Schwarzschild black hole, while if we set $\beta \rightarrow \infty$, then we obtain the metric function given by Eq. (17).

It should be pointed out that the curvature invariants, namely

$$
\begin{aligned}
R= & (2 \beta+1) \frac{(4 \beta M)^{2 \beta+1} q^{4}}{\left(4 \beta M r+q^{2}\right)^{2 \beta+1}} r^{2 \beta-3} \\
R_{\mu \nu} R^{\mu \nu}= & \frac{2^{(8 \beta+3)}(\beta M)^{4 \beta+2} q^{4}}{\left(4 \beta M r+q^{2}\right)^{4(\beta+1)}} \\
& \times\left[128 \beta^{2} M^{2} r^{2}-16 \beta(2 \beta-3) M q^{2} r+(4 \beta(\beta-1)+5) q^{4}\right] r^{4 \beta-6} \\
R_{\kappa \lambda \mu \nu} R^{\kappa \lambda \mu \nu}= & 2^{4(2 \beta+1)} M^{2}\left(\frac{\beta^{2} M^{2}}{\left(4 \beta M r+q^{2}\right)^{2}}\right)^{2 \beta}\left[1+\frac{\left(4 \beta M r-(2 \beta-1) q^{2}\right)^{2}}{\left(4 \beta M r+q^{2}\right)^{2}}\right. \\
& \left.+\frac{\left(16 \beta^{2} M^{2} r^{2}-8 \beta(2 \beta-1) M q^{2} r^{2}+(\beta(2 \beta-3)+1) q^{4}\right)^{2}}{\left(4 \beta M r+q^{2}\right)^{4}}\right] r^{4 \beta-6}
\end{aligned}
$$

are all regular everywhere if $\beta \geq 3 / 2$.

On the other hand, the WEC requires that the energy-momentum tensor must satisfy $T_{\mu \nu} t^{\mu} t^{\nu} \geq 0$ for all timelike vectors $t^{\mu}$ [41]. In general, in terms of the distribution functions $\sigma(r)$, the WEC implies (see also Eqs. (8) and (9) in Ref. [16])

$$
\frac{\sigma^{\prime}(r)}{r^{2}} \geq 0
$$

and

$$
2 \frac{\sigma^{\prime}(r)}{r} \geq \sigma^{\prime \prime}(r)
$$

It is evident that if we set $\beta \leq 3 / 2$ in Eq. (39) then the WEC is satisfied. Therefore, if we impose $\beta=3 / 2$, then we can construct a regular black hole solution which satisfies the WEC and of which the metric function is of the form

$$
f(r)=1-\frac{2 M}{r}\left(\frac{1}{1+\frac{q^{2}}{6 M r}}\right)^{3} .
$$

In addition, this black hole solution asymptotically behaves as the Reissner-Nordström solution. Note that the aforesaid metric function can also be obtained as in Ref. [16]. Moreover, it is known that if a charged regular black hole satisfies the WEC then such a regular black hole has de Sitter behavior at $r \rightarrow 0$. Thus, if we set $r \rightarrow 0$, then the metric function given by Eq. (45) behaves like 


$$
f(r) \approx 1-432 \frac{M^{4}}{q^{6}} r^{2}
$$

It should be stressed that the location of the horizons, i.e., $r_{ \pm}$, of the metric function given by Eq. (45) can be obtained by solving the following cubic equation:

$$
r^{3}+\left(\frac{q^{2}}{2 M}-2 M\right) r^{2}+\frac{q^{4}}{12 M^{2}} r+\frac{q^{6}}{216 M^{3}}=0 .
$$

It can be shown that if $q<4 M / 3$ then Eq. (47) has two positive real roots. The extremal regular black hole is obtained when $q_{\text {ext }}=4 M / 3$, and then Eq. (47) has one positive real root of the form

$$
r_{h \mathrm{ext}}=\frac{16 M}{27} .
$$

Furthermore, the electric field related to metric function given by Eq. (45) is written as

$$
E(r)=\frac{q}{r^{2}}\left(\frac{1}{1+\frac{q^{2}}{6 M r}}\right)^{5}
$$

which asymptotically becomes that of the Maxwell theory.

\section{More regular black hole metrics that satisfy the WEC}

In this section, we will construct new black hole metrics using distribution functions different from those used in previous sections. These black hole metrics will depend on the arbitrary parameter $\beta$, and we will determine the value of $\beta$ in such a way that regularity and the WEC will be satisfied.

We start by considering the standard Cauchy distribution function

$$
\sigma(x)=\frac{1}{\left(1+x^{2}\right)}
$$

and then we will make the change $x \rightarrow 1 / r$ with the appropriate factors. Thus, by substituting the above distribution function in Eq. (26) and setting $\beta=3 / 2$, we obtain a black hole metric which is regular everywhere and which also satisfies the WEC. It should be noted that this metric corresponds to the Bardeen solution, of which the mass function reads

$$
m(r)=\frac{M r^{3}}{\left(r^{2}+g^{2}\right)^{3 / 2}}=M\left(\frac{1}{1+g^{2} / r^{2}}\right)^{3 / 2}
$$

with $g$ being a self-gravitating magnetic monopole charge [15].

Next, we construct other black hole metrics based on the Dagum distribution [42], which is given by 


$$
\sigma(x)=\frac{a p x^{a p-1}}{b^{a p}\left(1+(x / b)^{a}\right)^{p+1}},
$$

with the variable $x>0$ and the parameters $a, b, p>0$.

First, it is easily seen that if we choose $a=3, b=1$ and $p=1 / 3$ we can build a new metric function by replacing Eq. (52) in Eq. (26) and of course we have to make the change $x \rightarrow 1 / r$ with the appropriate factors. In this case, when we set $\beta=3 / 4$, the black hole metric becomes regular everywhere and also satisfies the WEC. This corresponds to the black hole solution given in Ref. [20], of which the mass function reads

$$
m(r)=\frac{M}{1+2 l^{2} M / r^{3}}
$$

where $l>0$ is the Hubble length.

Second, if we choose $p=1 / a$ and $b=1$, then we can generalize the previous case with the following distribution function:

$$
\sigma(x)=\frac{1}{\left(1+x^{a}\right)^{\frac{a+1}{a}}} .
$$

Thus, making the change $x \rightarrow q^{2} /(M r)$ and selecting the appropriate factors, we can write the black hole metric in the form

$$
f(r)=1-\frac{2 M}{r}\left(\frac{1}{\left(1+\gamma\left(\frac{q^{2}}{M r}\right)^{a}\right)^{\frac{a+1}{a}}}\right)^{\beta}
$$

where $\gamma>0$. As before, we can calculate the curvature invariants and conclude that if we set $\beta \geq 3 /(a+1)$, then the black hole metric is regular everywhere; while if we set $\beta \leq 3 /(a+1)$, it satisfies the WEC. Thus, if we impose $\beta=3 /(a+1)$, we have a regular black hole metric which also satisfies the WEC and is written as

$$
f(r)=1-\frac{2 M}{r}\left(\frac{1}{1+\gamma\left(\frac{q^{2}}{M r}\right)^{a}}\right)^{3 / a} .
$$

The expression of the corresponding electric field is of the form

$$
E=\frac{q}{r^{2}}\left(\frac{3 \gamma(3+a)\left(\frac{q^{2}}{M r}\right)^{a-1}}{2\left(1+\gamma\left(\frac{q^{2}}{M r}\right)^{a}\right)^{2+3 / a}}\right) .
$$

The metrics that can be derived from Eq. (56) asymptotically behave as the Schwarzschild black hole, and, for small $r$, they behave as the de Sitter black hole,

$$
f(r) \approx 1-\frac{2 M^{4}}{\gamma^{3 / a} q^{6}} r^{2}
$$


At this point, it should be pointed out that, except for the case where $a=1$ and $\gamma=1 / 6$ which is equivalent to case of Eq. (45), in all other cases the regular black hole metrics asymptotically do not behave as the Reissner-Nordström black hole. Furthermore, only in the case where $a=1$ and $\gamma=1 / 6$, we have a model of nonlinear electrodynamics which, in the weak field approximation, corresponds to the Maxwell theory.

\section{Regular black holes that asymptotically behave as the Reissner-Nordström solution}

In the previous section, we constructed a family of regular black hole metrics given by Eq. (56), which satisfy the WEC. However, as already noted, these black hole metrics asymptotically do not behave as the Reissner-Nordström black hole when we consider the case where $a \geq 2$. So, in this section, we consider two methods to build regular black hole solutions using Eq. (56), of which the electric fields will asymptotically behave as that of the Maxwell theory.

The first method is to write the new black hole metrics in the form

$$
f(r)=1-\frac{2 M}{r}\left(\frac{1}{1+\gamma\left(\frac{q^{2}}{M r}\right)^{a}}\right)^{3 / a} \frac{\sigma(r)}{\sigma(\infty)}
$$

where $a \geq 2, \gamma>0$ is a constant and $\sigma(r)$ can be any one of the distribution functions listed in Table 1. Note that the values that the charge $q$ can take in order for the black hole metric to have horizons depends on the values of $\gamma$.

As an example of the general expression given by Eq. (59), we consider the case in which the distribution function is of the form

$$
\sigma(r)=\frac{q^{2} /(M r)}{\exp \left(\frac{q^{2}}{M r}\right)-1},
$$

with $a=4$ and $\gamma=1$. Thus, we obtain the following regular black hole metric:

$$
f(r)=1-\frac{2 M}{r}\left(\frac{1}{1+\left(\frac{q^{2}}{M r}\right)^{4}}\right)^{3 / 4}\left(\frac{q^{2} /(M r)}{\exp \left(\frac{q^{2}}{M r}\right)-1}\right) .
$$

In this case, the electric field associated with the aforesaid metric function is given as

$$
E(r)=-\frac{M r^{3}}{2 q} \frac{d}{d r}\left(\frac{1}{r^{2}} \frac{d}{d r}\left(\left(\frac{1}{1+\left(\frac{q^{2}}{M r}\right)^{4}}\right)^{3 / 4}\left(\frac{q^{2} /(M r)}{\exp \left(\frac{q^{2}}{M r}\right)-1}\right)\right)\right),
$$

which corresponds to an electric field obtained from a nonlinear electrodynamics theory. This electric field is regular everywhere and asymptotically behaves as 


$$
E(r) \approx \frac{q}{r^{2}}
$$

It is noteworthy that when $|q|=0.907 M$ we obtain the metric and electric field for the corresponding extremal regular black hole solution.

Furthermore, another interesting example which can be classified within the family of metrics described by Eq. (59) was developed in Ref. [12], by considering the distribution function

$$
\sigma(r)=\exp \left(\frac{q^{2}}{M r}\right)
$$

together with the Bardeen model, i.e., choosing $a=2$ and $\gamma=M^{2} / q^{2}$ in Eq. (56).

It should be stressed that all regular black hole metrics derived from the Eq. (59) asymptotically behave as the Reissner-Nordström black hole metric. However, these solutions do not satisfy the WEC.

The second method to build more regular black hole metrics based on those given by Eq. (56) consists of adding a new term which will make the metrics to behave asymptotically as the Reissner-Nordström metric. To build this new term we employ once again the distribution function of Dagum with a factor $q / r^{2}$. Thus, the metric function is written

$$
f(r)=1-\frac{2 M}{r}\left(\frac{1}{1+\gamma\left(\frac{q^{2}}{M r}\right)^{a}}\right)^{3 / a}+\frac{q^{2}}{r^{2}}\left(\frac{1}{1+\gamma\left(\frac{q^{2}}{M r}\right)^{a}}\right)^{4 / a},
$$

where $a \geq 2$ is an integer and $\gamma>0$ is a constant. It should be pointed out that if we set $\gamma \geq(2 / 3)^{a}$ then the associated solution satisfies the WEC. This can be demonstrated by calculating the corresponding distribution functions and their derivatives and then implementing Eqs. (43) and (44). The expression for the electric field associated with the above-mentioned metric is given by

$$
E=\frac{q}{r^{2}}\left(\frac{3 \gamma(3+a)\left(\frac{q^{2}}{M r}\right)^{a-1}}{2\left(1+\gamma\left(\frac{q^{2}}{M r}\right)^{a}\right)^{2+3 / a}}+\frac{1-\gamma(3+a)\left(\frac{q^{2}}{M r}\right)^{a}}{\left(1+\gamma\left(\frac{q^{2}}{M r}\right)^{a}\right)^{2(2+a) / a}}\right) .
$$

For small $r$, the black hole metrics obtained from Eq. (65) behave as the de Sitter black hole metric, namely

$$
f(r) \approx 1-\frac{M^{4}}{\gamma^{4 / a} q^{6}}\left(2 \gamma^{1 / a}-1\right) r^{2}
$$

Note that the factor that accompanies the term $r^{2}$ cannot become zero because of the restriction $\gamma \geq(2 / 3)^{a}$. On the other hand, if we set $(1 / 2)^{a} \leq \gamma<(2 / 3)^{a}$, the black hole metrics remain regular, without satisfying the WEC, but they have a de Sitter center. Therefore, if a regular black hole metric is regular and satisfies the WEC, then it has a de Sitter center [4]. However, if the metric has a de Sitter behavior when approaching the center, it does not necessarily satisfy the WEC. Furthermore, if we set $\gamma<(1 / 2)^{a}$, the black hole metric is not regular. 
Finally, there is a known case which can be obtained as a particular case of Eq. (65) by choosing $a=2$ and $\gamma=M^{2} / q^{2}$. This case corresponds to the black hole metric given in Ref. [8].

\section{Conclusions}

We have given various examples of regular black hole solutions that asymptotically behave as the Reissner-Nordström solution, and some of them also satisfy the weak energy condition. To construct regular black hole metrics, we employed several probability distribution functions in which we have replaced the variable by its reciprocal. In addition, we have also used powers of these distribution functions as a second method to construct new regular black hole metrics.

It is worth noting the case based on log-logistic distribution in the Sec. 5, where we have adjusted the power of the distribution function to get a black hole metric which is regular everywhere and also satisfies the WEC. One virtue of this solution is that the corresponding extremal case can be algebraically manipulated. Thus, we can revisit the aspects studied in Refs. [26], [27], and [28] by using this solution.

\section{Acknowledgments}

L.B. would like to thank Dirección de Investigación y Postgrado de la Universidad de La Frontera (DIUFRO).

\section{A Continuous probability distributions}

Some probability distributions which satisfy $\sigma(x)>0$ and $d \sigma(x) / d x<0$ for $x \geq 0$ are listed here (see, e.g., Ref. [43]).

The exponential distribution is

$$
\sigma(x)=e^{-x}
$$

The Fermi-Dirac distribution is

$$
\sigma(x)=\frac{1}{e^{x}+1} .
$$

The logistic distribution is

$$
\sigma(x)=\frac{e^{-x}}{\left(1+e^{-x}\right)^{2}} .
$$

The hyperbolic secant distribution is [44]

$$
\sigma(x)=\frac{2}{e^{x}+e^{-x}} .
$$

The Einstein functions are 


$$
\sigma(x)=\frac{x}{e^{x}-1}
$$

and

$$
\sigma(x)=\frac{x^{2} e^{x}}{\left(e^{x}-1\right)^{2}}
$$

\section{B Dual $\mathbf{P}$ formalism}

We show here the description based in a dual representation of nonlinear electrodynamics obtained by a Legendre transformation [40].

The metric function and its corresponding electromagnetic field arise as a solution of Einstein field equations coupled to a nonlinear electrodynamics model; that is,

$$
S=\int d^{4} x \sqrt{-g}\left(\frac{1}{16 \pi} R-\frac{1}{4 \pi} L(F)\right)
$$

where $R$ is the scalar curvature and the Lagrangian $L$ depends on $F=\frac{1}{4} F_{\mu \nu} F^{\mu \nu}$ which, for weak fields, describes the Maxwell theory. One can describe the system under study in terms of an auxiliary field defined by $P_{\mu \nu}=(d L / d F) F_{\mu \nu}$. The dual representation is obtained by means of a Legendre transformation

$$
H=2 F \frac{d L}{d F}-L
$$

which is a function of the invariant $P=\frac{1}{4} P_{\mu \nu} P^{\mu \nu}$. Thus, we can express the Lagrangian $L$ depending on $P_{\mu \nu}$ as

$$
L=2 P \frac{d H}{d P}-H
$$

and the electromagnetic field as

$$
F_{\mu \nu}=\frac{d H}{d P} P_{\mu \nu} .
$$

The energy-momentum tensor in the dual representation is written as

$$
T_{\mu \nu}=\frac{1}{4 \pi} \frac{d H}{d P} P_{\mu \alpha} P_{\nu}^{\alpha}-\frac{1}{4 \pi} g_{\mu \nu}\left(2 P \frac{d H}{d P}-H\right) .
$$

It follows from the components of $T_{\mu \nu}$ that $M^{\prime}(r)=-r^{2} H(P)$. Therefore, we can obtain the corresponding mass function.

We now list the function $H(P)$ for each distribution given in Table 1, and to simplify notation we define $U=s \sqrt[4]{-2 q^{2} P}, s=q /(2 M)$.

Exponential:

$$
H=P e^{-U}
$$


Fermi-Dirac:

$$
H=4 P \frac{e^{2 U}}{\left(1+e^{2 U}\right)^{2}}=P\left(1-\tanh ^{2}(U)\right)
$$

Logistic:

$$
H=\frac{P}{\sqrt{U}} \operatorname{sech}^{2} \sqrt{U} \tanh \sqrt{U}
$$

Hyperbolic secant:

$$
H=\frac{P}{\sqrt{2 U}} \operatorname{sech} \sqrt{2 U} \tanh \sqrt{2 U}
$$

Einstein:

$$
\begin{gathered}
H=P\left(4 U e^{2 U}-2\right) \frac{1}{e^{2 U}-1}, \\
H=3 P \operatorname{csch}^{2}(\sqrt{3 U})(\sqrt{3 U} \operatorname{coth}(\sqrt{3 U})-1) .
\end{gathered}
$$

Finally, we also include the case inspired by the log-logistic distribution which reads

$$
H=\frac{P}{(1+U / 3)^{4}} .
$$

\section{References}

[1] S. Ansoldi, arXiv:0802.0330 [gr-qc].

[2] E. Elizalde and S. R. Hildebrandt, Phys. Rev. D 65, 124024 (2002).

[3] O. B. Zaslavskii, Phys. Lett. B 688, 278 (2010).

[4] I. Dymnikova, Class. Quant. Grav. 19, 725 (2002).

[5] N. Breton, Gen. Rel. Grav. 37, 643 (2005).

[6] S. Bose and N. Dadhich, Phys. Rev. D 60, 064010 (1999).

[7] L. Balart, Phys. Lett. B 687, 280 (2010).

[8] E. Ayon-Beato and A. Garcia, Phys. Rev. Lett. 80, 5056 (1998).

[9] E. Ayon-Beato and A. Garcia, Gen. Rel. Grav. 37, 635 (2005).

[10] I. Dymnikova, Class. Quant. Grav. 21, 4417 (2004).

[11] E. Ayon-Beato and A. Garcia, Phys. Lett. B 464, 25 (1999).

[12] E. Ayon-Beato and A. Garcia, Gen. Rel. Grav. 31, 629 (1999).

[13] K. A. Bronnikov, Phys. Rev. D 63, 044005 (2001). 
[14] J. M. Bardeen, in Proceedings of GR5, Tbilisi, U.S.S.R, p.174 (1968).

[15] E. Ayon-Beato and A. Garcia, Phys. Lett. B 493, 149 (2000).

[16] L. Balart and E. C. Vagenas, Phys. Lett. B 730, 14 (2014).

[17] P. Nicolini, A. Smailagic and E. Spallucci, Phys. Lett. B 632, 547 (2006).

[18] S. Ansoldi, P. Nicolini, A. Smailagic and E. Spallucci, Phys. Lett. B 645, 261 (2007).

[19] I. Dymnikova, Gen. Rel. Grav. 24, 235 (1992).

[20] S. A. Hayward, Phys. Rev. Lett. 96, 031103 (2006).

[21] J. P. S. Lemos and V. T. Zanchin, Phys. Rev. D 83, 124005 (2011).

[22] E. F. Eiroa and C. M. Sendra, Class. Quant. Grav. 28, 085008 (2011).

[23] Y. S. Myung, Y. W. Kim and Y. J. Park, Phys. Lett. B 656, 221 (2007).

[24] W. J. Mo, R. G. Cai and R. K. Su, Commun. Theor. Phys. 46, 453 (2006).

[25] Y. S. Myung, Y. W. Kim and Y. J. Park, Gen. Rel. Grav. 41, 1051 (2009).

[26] J. Matyjasek, Phys. Rev. D 70, 047504 (2004).

[27] Y. S. Myung, Y. W. Kim and Y. J. Park, Phys. Lett. B 659, 832 (2008).

[28] Y. S. Myung, Y. W. Kim and Y. J. Park, Phys. Rev. D 76, 104045 (2007).

[29] J. Matyjasek, D. Tryniecki and M. Klimek, Mod. Phys. Lett. A 23, 3377 (2008).

[30] J. Matyjasek, Phys. Rev. D 63, 084004 (2001).

[31] W. Berej and J. Matyjasek, Phys. Rev. D 66, 024022 (2002).

[32] J. Matyjasek, Phys. Rev. D 76, 084003 (2007).

[33] W. Berej, J. Matyjasek, D. Tryniecki and M. Woronowicz, Gen. Rel. Grav. 38, 885 (2006).

[34] J. Matyjasek, Acta Phys. Polon. B 39, 3 (2008).

[35] F. S. N. Lobo and A. V. B. Arellano, Class. Quant. Grav. 24, 1069 (2007).

[36] L. Hollenstein and F. S. N. Lobo, Phys. Rev. D 78, 124007 (2008).

[37] C. Moreno and O. Sarbach, Phys. Rev. D 67, 024028 (2003).

[38] N. Breton, Phys. Rev. D 72, 044015 (2005). 
[39] N. Breton and S. E. P. Bergliaffa, arXiv:1402.2922 [gr-qc].

[40] I. H. Salazar, A. Garcia and J. Plebanski, J. Math. Phys. 28, 2171 (1987).

[41] S. W. Hawking and G. F. R. Ellis, The Large Scale Structure of Space-Time (Cambridge University Press, Cambridge, England, 1973).

[42] C. Dagun, Economie Appliquée. 30, 413 (1977).

[43] E. W. Weisstein, CRC Concise Encyclopedia of Mathematics (CRC Press, Boca Raton, Florida, 1999).

[44] W. D. Baten, Bull. Amer. Math. Soc. 40, 284 (1934). 